\title{
An Uncommon Presentation of a Rare Disorder - Ruptured Vein of Galen Malformation with Atrial Septal Defect
}

\begin{abstract}
Vein of Galen aneurysmal malformations (VGAMs) are rare intracranial anomalies constituting $1 \%$ of all the cerebrovascular malformations. Its association with congenital heart disease has been described. Most reported cases are in the postnatal period associated with cardiac failure. Rupture of this malformation is uncommon. Here we describe a case of an otherwise healthy 25 year old presenting with sudden change in his mental status. Unenhanced CT brain showed intracranial hemorrhage secondary to rupture of the VGAM. Cerebral angiography showed stenosis of a persistent falcine sinus, which likely delayed his presentation. Endovascular embolization of the varix was performed. Echocardiogram showed a large sinus venosus ASD with anomalous pulmonary venous return. He sustained residual neurological deficits and is currently undergoing rehabilitation. This is a rare case of delayed presentation of this disease despite the underlying cardiac defect, with an uncommon complication of rupture and an unfortunate outcome.
\end{abstract}

Keywords: Vein of Galen aneurysmal Malformations; Persistent falcine sinus; Sinus venosus ASD; Intracranial bleed; Endovascular embolisation

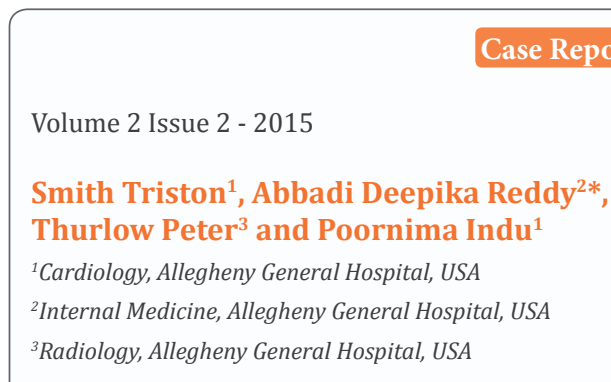

*Corresponding author: Abbadi Deepika, Allegheny General Hospital, 320 E North Ave, Pittsburgh, PA- 15212 USA, Tel: 4123594971; Fax: 4123594983; Email: dabbadi@wpahs.org

Received: January 19, 2015 | Published: March 13, 2015
Abbreviations: VGAMs: Vein of Galen Aneurysmal Malformations; ASD: Atrial Septal Defect; SVC: Superior Vena Cava; EVD: ExternalVentricular Drains; VP:Ventriculo-Peritoneal; AVM: Arteriovenous Malformations; AVF: Arteriovenous Fistula; VOG: Vein of Galen; PCA: Posterior Cerebral Artery; MCA: Middle Cerebral Artery

\section{Introduction}

VGAMs commonly manifest in the first year of birth with high output cardiac failure and have a very high mortality $[1,2]$. A continuous murmur at the base of the skull can be heard in $80 \%$ of the newborns due to the AV fistula. It can also be detected on in utero Ultrasonography in the third trimester of pregnancy [3]. Endovascular embolization of the feeding vessels plays a key role in the treatment of VGAMs and is a primary determinant of neurological outcomes [4]. We present a rare manifestation of this disease with delayed onset that reinforces the importance of early detection and treatment of this deadly malformation to prevent catastrophic outcomes.

\section{Case Presentation}

A 25-year-old male with no past medical history presented with sudden onset of headache, vomiting and right-sided weakness while watching television. His vital signs were normal and stable on arrival to the emergency department. On physical examination he was somnolent but easily aroused and oriented, although his speech was slurred. The right pupil was $2 \mathrm{~mm}$ in diameter with sluggish reaction to light but the left pupil diameter was $4 \mathrm{~mm}$ and reactive. Laboratory investigations were unremarkable except for a lactic acid of $3.3 \mathrm{mmol} / \mathrm{L}$. Unenhanced CT of the brain was performed showing ectatic blood vessels surrounding a dilated venous varix in the region of the Vein of Galen (Figure 1a). Peripheral calcifications within the dilated vein as well as interventricular and subarachnoid hemorrhages were also seen noted (Figure $1 \mathrm{~b}$ ).

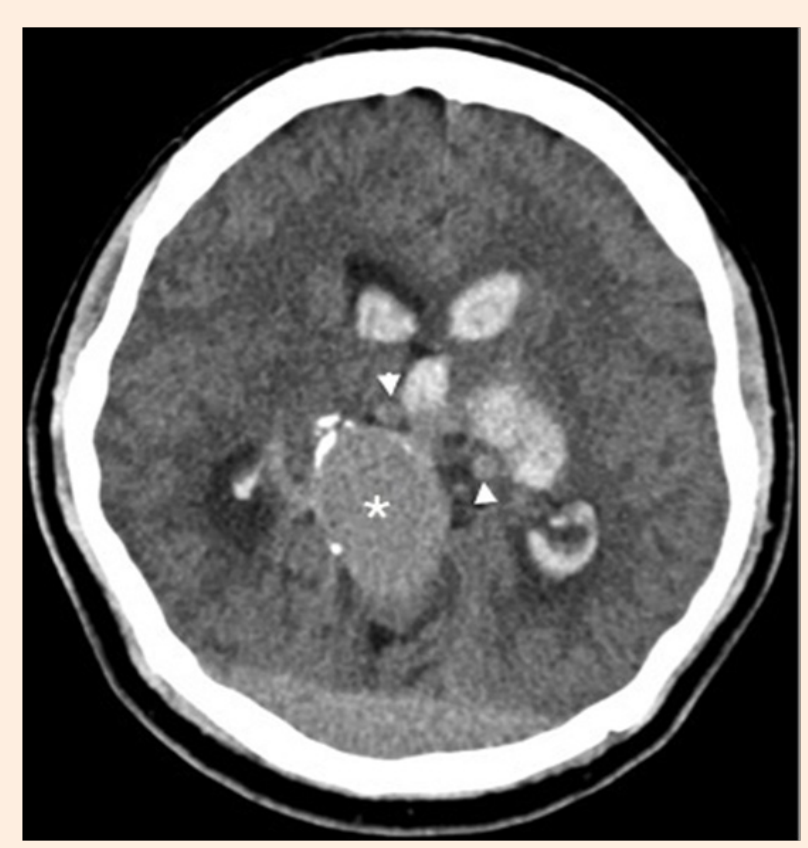

Figure 1a: Unenhanced CT of the brain. Ectatic blood vessels (arrowheads) surround a dilated venous varix (asterisk) in the region of the vein of Galen and straight sinus. 


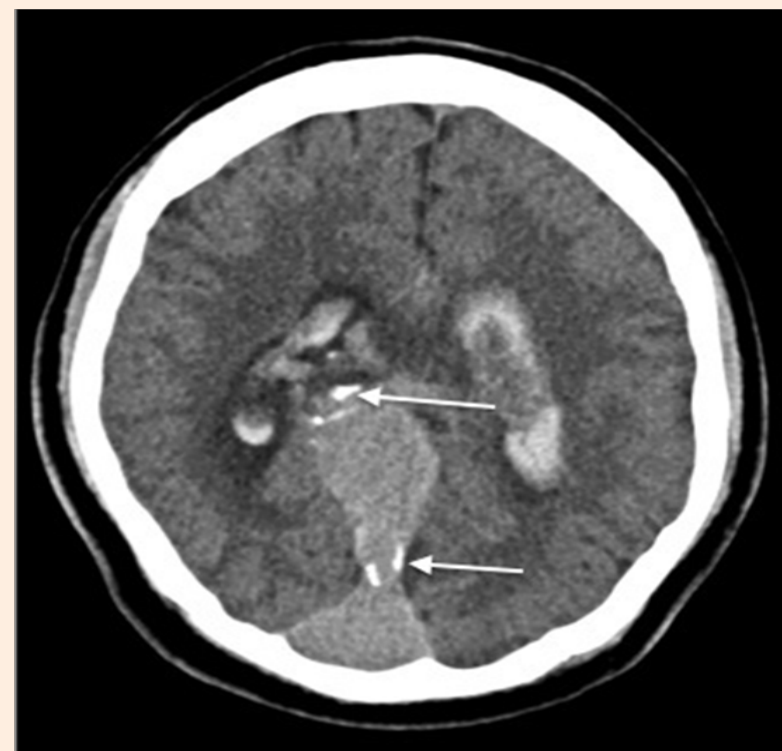

Figure 1b: Unenhanced CT of the brain. Peripheral calcification within the dilated vein, suggesting high flow venopathy secondary to arteriovenous shunting. Parenchymal, intraventricular and subarachnoid hemorrhage with hydrocephalus is also present. These findings are consistent with a ruptured vein of Galen malformation (VGAM).

The patient was started on an intravenous infusion of Mannitol and 3\% saline for impending herniation. His mental status gradually worsened in the emergency department and was intubated for airway protection. Digital subtraction angiography was subsequently done clearly demonstrating the presence of a large choroidal type vein of Galen malformation with the particular features illustrated in (Figures 2a \& 2b). 24 hrs after admission the patient became febrile. Blood cultures were positive for enterobacter. Transesophageal echocardiogram performed to evaluate for the presence of valvular vegetations revealed the presence of a large sinus venosus defect $(11 \mathrm{~mm})$ with a large right to left shunt demonstrated with agitated saline contrast injection and color Doppler (Figures 3a \& 3b). An anomalous right superior pulmonary vein draining into the SVC, which has an association with sinus venosus ASD, was also detected.

\section{Treatment}

Endovascular therapy with onyx (n-butyle-2-cyanoacrylate) embolization to the main feeder from the right PCA was done to decrease the shunting. External ventricular drains (EVDs) were placed to help manage the hydrocephalus. Serial CT's of the brain were done to monitor hydrocephalus and shunts. He continued to be ventilator dependent and was initially on levetiracetam for seizure prophylaxis. This was switched to pentobarbital to induce coma, and serial EEG's monitoring performed to assess for seizure activity. During the course of his treatment the patient developed a pulmonary embolism but could not be anti coagulated secondary to the intracranial bleed. An inferior vena cava filter was therefore placed.

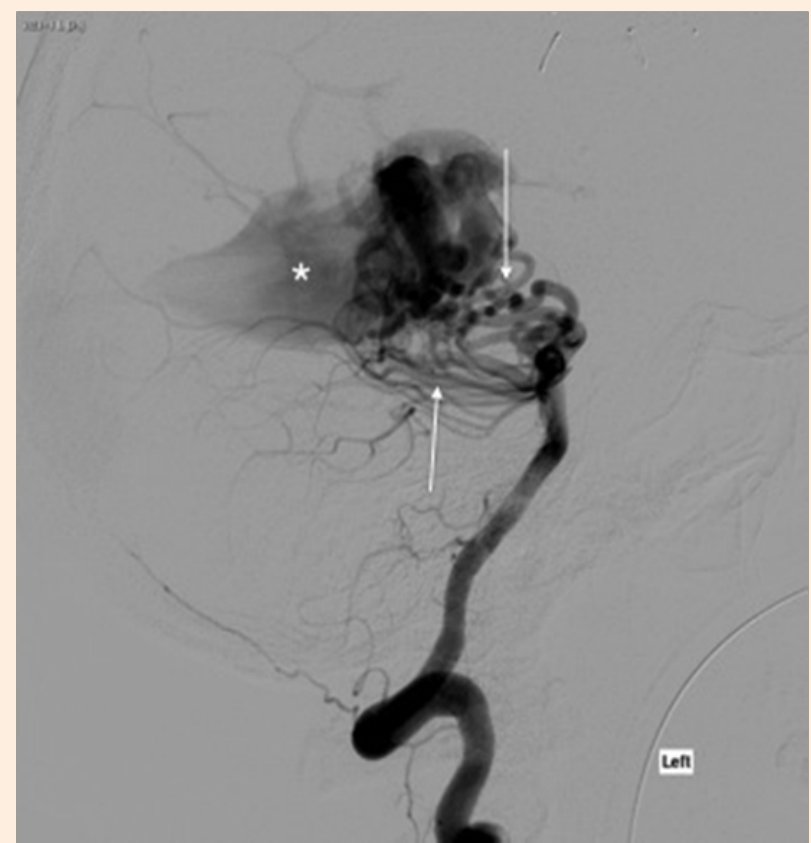

Figure 2a: Digital subtraction angiography (DSA). Lateral DSA following left vertebral artery injection demonstrates a large, choroidal-type vein of Galen malformation (VGAM). Arteriovenous shunting from thebilateral posterior cerebral arteries (PCA) through numerous ectatic posterior choroidal and thalamoperforating arteries (arrows) results in early filling of a dilated median prosencephalic vein (MPV) ofMarkowski (asterisk).

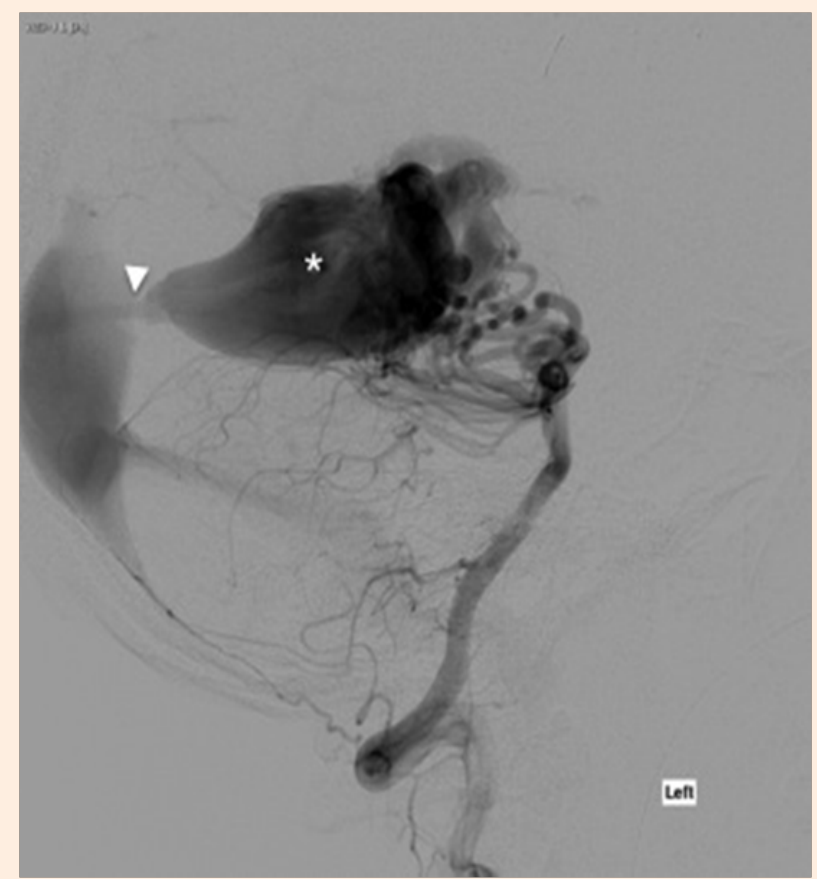

Figure 2b: Digital subtraction angiography (DSA). Lateral DSA following left vertebral artery injection demonstrates a large, choroidal-type vein of Galen malformation (VGAM). The dilated MPV drains through apersistent falcine sinus into a dilated distal superior saggital sinus and torcula. High flow venopathy results in a stenosis (arrowhead) of the distal falcine sinus. 


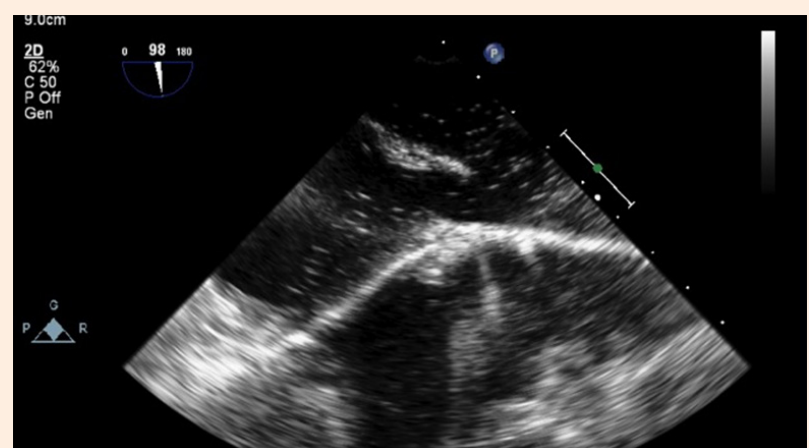

Figure 3a: TEE images showing positive bubble study.

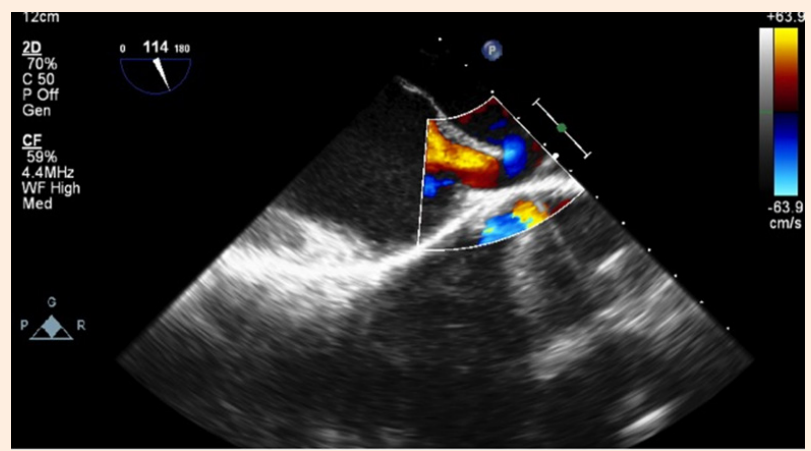

Figure 3b: Color Doppler consistent with Sinus Venosus ASD with bidirectional shunt.

\section{Outcome and Follow-Up}

The patient could not be weaned off the ventilator. He had a tracheostomy and percutaneous endoscopic gastrostomy (PEG) tube placed. He couldn't be taken off the EVD's as weaning caused worsening of mental status and increasing hydrocephalus. Hence a ventriculoperitoneal (VP) shunt was placed and he was discharged to a skilled nursing facility after a 40-day hospital stay. Unfortunately, he has been lost to follow up at our institution.

\section{Discussion}

Vein of Galen (VOG), named after its discover, the Greek physician Galen, drains blood from the inferior sagittal sinus and internal cerebral veins into the straight sinus. VGAMs are not true aneurysms. They comprise a group of aneurysmally dilated midline deep venous structures that represent the median prosencephalic vein, and they share dilation of the VOG in common $[5,6]$. They are fed by abnormal arteriovenous communications usually from one or both PCA, as in our patient, and less frequently from small posterior branches of MCA and other arteries [5,7]. Angioarchitectural analyses classifies them into 5 types such as parenchymatous AVMs (44\%), mural AVF (20\%), choroidal AVF (30\%), dural AV fistulas (3\%), and Vein of Galen varices $(7 \%)[1,8]$. Choroidal type seen in this patient commonly presents in the new born. Mural and parenchymal forms are smaller and have later onset between 1-15 months of age $[4,8]$.

Cases of VGAMs associated with congenital heart disease have been reported in the literature, the first being reported in 1994 [9]. Atrial septal defects (ASD) constitute $5 \%$ of these congenital defects. The sinus venosus type with anomalous drainage of the pulmonary veins into the superior vena cava (SVC) or right atrium (RA) is seen in only $5-10 \%$ of ASD cases $[2,9,10]$. It is hypothesized that increased SVC return in utero from the AVM may interfere with the absorption of the right horn of sinus venosus into the RA, which is completed around 8 weeks [9]. The VOG also develops around the same period between 6-11 weeks. In some cases the superior vena cava can override the septal defect promoting direct drainage of venous return into the left atrium, thus facilitating paradoxical embolization [11]. VAGMs are most commonly detected in utero or after birth because exclusion of the low resistance placental flow causes an abrupt increase in flow across the fistula and almost $80 \%$ of cardiac output is diverted to brain in severe cases. This causes a compensatory increase in cardiac output and blood volume to maintain systemic perfusion leading to its most common presentation, high output cardiac failure $[10,11]$.

If untreated, mortality approaches $95 \%$ in the $1^{\text {st }}$ month and $33 \%$ between 1-12 months of age. Manifestations in childhood include intracranial bleed, subarachnoid hemorrhage, myocardial ischemia, pulmonary hypertension, varying degrees of neurologic deficits and developmental delay $[1,3,5,10]$. Progressive hydrocephalus, secondary to compression of the aqueduct, and high flow venopathy can cause occlusion or stenosis of the distal venous drainage system as in this case. Periventricular infarction, calcification and thrombosis of the aneurysmal vein can also occur (our case). It is rare for patients to have their first manifestation of this disorder in the third decade of life. VAGM is described as the "Gordon knot" of cerebrovascular system and complete elimination is rarely achieved. Endovascular therapy with embolization is the main stay and often requires several successive procedures [1,3].

A 30-month follow-up study reported that 55\% of their patients were functionally normal and more recent studies report $9-15 \%$ mortality and little to no neuromorbidity in 61 $66 \%$ of survivors $[1,7,8]$. The cardiac defects should be looked for and managed later if they continue to be in heart failure despite treating the VAGM. Hybrid periatrial device closure of the ASD can be done before neurological intervention, to avoid cardiopulmonary bypass ad eliminate intracardiac embolic risk during treatment of VAGM. In summary this patient could have had a delayed presentation with an uncommon presentation of rupture secondary to the narrowing of the persistent falcine sinus, which is rare as well. His cardiac defect could have been masked too because of the same reason. As this presentation had a catastrophic outcome, it is prudent to detect these early in life even with a slight suspicion and treat them aggressively.

\section{References}

1. Recinos PF, Rahmathulla G, Pearl M, Recinos VR, Jallo GI, et al. (2012) Vein of Galen malformations: epidemiology, clinical presentations, management. Neurosurg Clin N Am 23(1): 165-177.

2. McElhinney DB, Halbach VV, Silverman NH, Dowd CF, Hanley FL (1998) Congenital cardiac anomalies with vein of Galen malformations in infants. Arch Dis Child 78(6): 548-551.

3. Sasidharan CK, Anoop P, Vijayakumar M, Jayakrishnan MP, Reetha G, et al. (2004) Spectrum of clinical presentations of vein of Galen aneurysm. Indian J Pediatr 71(5): 459-463. 
4. Gailloud P, O’Riordan DP, Burger I, Levrier O, Jallo G, et al. (2005) Diagnosis and management of vein of galen aneurysmal malformations. J Perinatol 25(8): 542-551.

5. Alvarez H, Monaco GR, Rodesch G, Sachet M, Krings T, et al. (2007) Vein of galen aneurysmal malformations. Neuroimaging Clin $\mathrm{N}$ Am 17(2): 189-206.

6. Meila D, Paramasivam S, Niimi Y, Brassel F, Berenstein A, et al (2012) The persistent primitive marginal sinus-observations in children with Vein of Galen malformation. Neuroradiology 54(12): 1375-1379.

7. Cai MJ, Ma LT, Yang M, Pan L (2012) Vein of Galen aneurysmal malformation: strategy transarterial embolization using coils alone. Neurol India 60(5): 559-560.
8. Lasjaunias P, Rodesch G, Terbrugge K, Pruvost P, Devictor D, et al. (1989) Vein of Galen Aneurysmal Malformations Report of 36 Cases Managed Between 1982 and 1988. Acta Neuroehir (Wien) 99(1-2): 26-37.

9. Friedman DM, Rutkowski M, Madrid M, Berenstein A (1994) Sinus Venosus Atrial Septal Defect Associated with Vein of Galen Malformations: Report of Two Cases. Pediatr Cardiol 15(1): 50-52.

10. Patel N, Mills JF, Cheung MM, Loughnan PM (2007) Systemic haemodynamics in infants with vein of Galen malformation: assessment and basis for therapy. J Perinatol 27(7): 460-463.

11. Ahmad NS, Walker IA, Brew S (2008) Repair of sinus venosus defect in a patient with untreated vein of Galen aneurysmal malformation. Paediatr Anaesth 18(6): 548-553. 